\title{
Optimization of a k-covering of a bounded set with circles of two given radii
}

https://doi.org/10.1515/comp-2020-0219

Received Oct 28, 2019; accepted Dec 10, 2020

\begin{abstract}
A numerical method for investigating $k$-coverings of a convex bounded set with circles of two given radii is proposed. Cases with constraints on the distances between the covering circle centers are considered. An algorithm for finding an approximate number of such circles and the arrangement of their centers is described. For certain specific cases, approximate lower bounds of the density of the $k$-covering of the given domain are found. We use either $0-1$ linear programming or general integer linear programming models. Numerical results demonstrating the effectiveness of the proposed methods are presented.
\end{abstract}

Keywords: Combinatorial optimization, $k$-covering with circles of two radii, Multiple coverings, Estimation of $k$ covering density with circles of two radii

\section{Introduction}

Let $G$ be a bounded convex closed set with a nonempty interior on the plane $P$, and let closed circles with the radii $r_{1}$ and $r_{2}$ be given. A set of these circles forms a $k$-covering $(k \geq 1)$ of the domain $G$ if each point $s$ of $G$ belongs to at least $k$ circles. The density of a covering of $G$ is defined as the ratio of the total area of the covering circles to the area of $G$.

Problems of covering a given domain with circles of either identical or different radii are important for applications, including emergency response system design, locating radio stations, and navigation facilities. Other many applications areas are given in [1-6]. There are a lot of applications investigating wireless sensor networks for monitoring a given domain when sensors have equal or different ranges. In a typical scenario, several different types of sen-

\footnotetext{
${ }^{\star}$ Corresponding Author: Alexander V. Khorkov: Kazan National Research Technical University named after A.N. Tupolev-KAI; Email: aLex22fcrk@yandex.ru

Shamil I. Galiev: Kazan National Research Technical University named after A.N. Tupolev-KAI; Email: sh.galiev@mail.ru
}

sors are available which can be appropriately placed in the given field [7-12]. Designing such a system requires solving the problem of finding a $k$-covering $(k \geq 1)$ of the given domain with circles of equal or different radii.

There are a lot of publications devoted to coverings with equal circles (see [13-16] and references therein). In studies devoted to coverings with equal circles, covering of specific domains: a square, a rectangle, a circle, and some others are considered. Covering problems for sets that do not coincide with whole plane, e.g., strips, are studied in papers [17-19], see also references therein. There are also publications in which $k$-coverings, $k \geq 1$, are studied (e.g., see $[10,12,20])$. Some results for determining the number of circles needed for a $k$-covering of certain given convex bounded closed domains are reported in [21].

The $k$-covering problem of a finite set with circles of different radii is studied in [22], where a nonlinear model of the problem is constructed that is then transformed into a linear problem with a large number of variables, auxiliary variables, and constraints. The variable radius covering problem is considered in [6]. Mathematical programming formulations are proposed for the discrete problem and a solution approach is suggested and tested. See [23] for covering the plane with two kinds of circles.

In this paper, we propose a method for investigating $k$ coverings of an arbitrary convex bounded closed set with a nonempty interior with circles of two given radii. Cases with constraints on the distances between the covering circle centers are considered. We use $0-1$ linear programming or general integer linear programming models. An algorithm for finding an approximate number of such circles and the arrangement of their centers is described. For certain specific cases, approximate lower bounds of the density of the $k$-covering of the given domain are found. Numerical results demonstrating the effectiveness of the proposed methods are presented. In some cases the obtained value of $k$-covering density coincides with the lower bound of the density of the $k$-covering, therefore, the coverings in these cases are not improvable; in other cases the difference between obtained value and lower estimates of the covering densities characterizes the quality of the covering.

๖ Open Access. @ 2021 A. V. Khorkov and Sh. I. Galiev, published by De Gruyter. Attribution 4.0 License 
In this paper, we use the results of [24-27], and some of them are given for completeness and clarity of presentation.

\section{Problem of Finding a k-covering of a Bounded Set with Circles of Two Radii}

Let $r_{1}>r_{2}$ be an assumption that holds throughout. We will choose $\Delta>0$ and build a rectangular grid with the step size $\Delta x=\Delta y=\Delta$ in $G$. The set of grid lines forming this set in $G$ generates the square cells $C_{\Delta}$ with the side $\Delta$. If such a cell lies entirely in $G$, then each of its vertex is a grid node. If the square $C_{\Delta}$ belongs to $G$ only, then each vertex $C_{\Delta}$ in $G$, the points at which the boundary of $G$ enters $C_{\Delta}$, and the points at which the boundary of $G$ exits $C_{\Delta}$ are considered as grid nodes. In addition, we add on the boundary $F$ of the domain $G \cap C_{\Delta}$ node points such that the distance between them along the line $F$ does not exceed $\Delta$. Let $T_{\Delta}$ consist of all grid points described above: $T_{\Delta}=\left\{t_{1}, \ldots, t_{n}\right\}$, where $t_{i} \in G$ and $1 \leq i \leq n$.

Instead of the rectangular grid on $G$, we can construct another grid, e.g., an oblique one. If $G$ is an equilateral triangle, then we construct the oblique grid as follows. Select a step $\Delta$ such that it exactly divides the triangle side length. Draw lines parallel to each side of the triangle with the step $\Delta$ along the triangle sides. As a result, these grid lines generate equilateral triangles $C_{\Delta}$ with the sides $\Delta$. The nodes of this grid form the set $T_{\Delta}$.

Below, if not otherwise specified, we assume that the grid is rectangular. Here and in what follows, $d(s, t)$ is the Euclidean distance between the points $s$ and $t$.

Let $G, r_{1}, r_{2}, k(k \geq 1)$, and $\Delta$ be given and $T_{\Delta}$ be constructed. Consider the following problems.

P1. Find a $k$-covering of the domain $G$ with circles of the given radii $r_{1}$ and $r_{2}$ centered in $G$ so as to minimize the total area of the covering circles and determine the location of their centers in $G$.

P2. Find a $k$-covering of the domain $G$ with circles of the given radii $r_{1}$ and $r_{2}$ so as to minimize the total area of the covering circles and determine the location of their centers under the condition that the circle centers are at the points of $T_{\Delta}$ and there is not more than one circle center at each point of $T_{\Delta}$.

P3. Find a $k$-covering of the set $T_{\Delta}$ with circles of the given radii $r_{1}$ and $r_{2}$ so as to minimize the total area of the cov- ering circles and determine the location of their centers under the condition that the circle centers are at the points of $T_{\Delta}$ and there is not more than one circle center at each point of $T_{\Delta}$.

P4. Find a $k$-covering of the domain $G$ with circles of the given radii $r_{1}$ and $r_{2}$ so as to minimize the total area of the covering circles and determine the location of their centers under the condition that the circle centers are at the points of $T_{\Delta}$.

P5. Find a $k$-covering of the set $T_{\Delta}$ with circles of the given radii $r_{1}$ and $r_{2}$ so as to minimize the total area of the covering circles and determine the location of their centers under the condition that the circle centers are at the points of $T_{\Delta}$.

\section{First, consider P3.}

It is easy to verify that a sufficient condition for the solvability of $\mathrm{P} 3$ is as follows:

For each point $t_{j}$ from the set $T_{\Delta}$ there exist at least $k-1$ different points $t_{i}\left(t_{i}\right.$ is not equal

to $t_{j}$ ) in $T_{\Delta}$ such that the distance from $t_{j}$ to these points does not exceed $r_{2}$.

Let us construct a mathematical model of P3. We assume that $\Delta$ has already been selected and the (finite) set $T_{\Delta}$ has been constructed. Introduce the coefficients $a_{i j}$ and $a_{i, n+j}$ where $1 \leq i, j \leq n$ :

$$
\begin{array}{r}
a_{i j}=\left\{\begin{array}{lll}
1, & \text { if } & d\left(t_{i}, t_{j}\right) \leq r_{1}, \\
0, & \text { if } & d\left(t_{i}, t_{j}\right)>r_{1},
\end{array}\right. \\
a_{i, n+j}=\left\{\begin{array}{lll}
1, & \text { if } & d\left(t_{i}, t_{j}\right) \leq r_{2}, \\
0, & \text { if } & d\left(t_{i}, t_{j}\right)>r_{2} .
\end{array}\right.
\end{array}
$$

Define the following variables: $z_{i}$ is the number of circle centers of radius $r_{1}$ located at the point $t_{i}, 1 \leq i \leq n$; $z_{n+j}$ is the number of circle centers of radius $r_{2}$ located at the point $t_{j}, 1 \leq j \leq n$. In the case of $k$-covering P2 and P3, it is clear that, at any point of $T_{\Delta}$ cannot contain more than one center of a circle of radius $r_{1}$ or $r_{2}$. Therefore, we have the constraints

$$
z_{i} \in\{0,1\}, 1 \leq i \leq 2 n .
$$

In addition, we have the constraints

$$
z_{i}+z_{i+n} \leq 1,1 \leq i \leq n .
$$

If the total area of the covering circles is minimized, then for $k=1$ constraints (4) are redundant. 
We formulate the problem

$$
\left(z_{1}+\ldots+z_{n}\right)+\left(\frac{r_{2}}{r_{1}}\right)^{2}\left(z_{n+1}+\ldots+z_{2 n}\right) \rightarrow \min
$$

subject to the constraints

$$
\begin{gathered}
a_{11} z_{1}+a_{12} z_{2}+\ldots+a_{1,2 n} z_{2 n} \geq k_{1}, \\
\ldots \\
a_{n 1} z_{1}+a_{n 2} z_{2}+\ldots+a_{n, 2 n} z_{2 n} \geq k_{n}, \\
z_{i} \in\{0,1\}, 1 \leq i \leq 2 n .
\end{gathered}
$$

Here $k_{j}$ is the prescribed multiplicity of covering of the point $t_{j}, 1 \leq j \leq n$. Constraints (6) ensure that each point $t_{j}$ in $T_{\Delta}$ is covered by at least $k_{j}$ circles. If $k_{j}=k$ for $1 \leq j \leq n$, then we have a $k$-covering of the set $T_{\Delta}$. If the variable $z_{i}$ is equal to one, then the point $t_{i}$ of $T_{\Delta}(1 \leq i \leq n)$ is the center of a circle of radius $r_{1}$. If $z_{n+i}$ is equal to one, then the point $t_{i}$ of $T_{\Delta}(1 \leq i \leq n)$ is the center of a circle of radius $r_{2}$.

Problem presented in relation (5)-(7), (4) is the problem of finding a $k$-covering of a finite set of points $T_{\Delta}$ in which the total area of the covering circles is minimal. As a result, we have a $0-1$ linear program of size $2 n$. Denote this problem by W1. It is known that the general problem W1 is NP-hard (see [28-33]).

Condition (1) for the solvability of $\mathrm{P} 3$, i.e., of problem $\mathrm{W} 1$, is difficult to verify. We give easily verifiable sufficient solvability conditions of W1 for some specific types of the set $G$ for the covering multiplicity not exceeding ten.

First, consider the case when $G$ is a square, which we will denote by $Q$. In $Q$, we construct a rectangular grid with a step $\Delta x=\Delta y=\Delta$. For simplicity, we assume that $\Delta$ exactly divides the square side length. Consider the covering of $Q$ with circles of radii $r_{1}$ and $r_{2}$. For a point $s$, denote by $R(s)$ the closed circle of radius $r_{2}$ centered at $s$. Let $s$ be a vertex of $Q$; then, $s$ coincides with a point of $T_{\Delta}$. Then, in the case $r_{2}=\Delta$ the set $Q \cap R(s)$ obviously contains three different points of $T_{\Delta}$; if $r_{2}=\Delta \sqrt{2}$, then the set $Q \cap R(s)$ contains four different points of $T_{\Delta}$, etc. For any other point $s$ in $T_{\Delta}$ (that is not a vertex of the square), the number of different points of $T_{\Delta}$ in $Q \cap R(s)$ is no less than this number in the case when $s$ is a vertex of the square. Therefore, if $1 \leq k \leq 3$, then $\Delta \leq r_{2}$; if $1 \leq k \leq 4$, then $\Delta \leq r_{2} / \sqrt{2}$; if $1 \leq k \leq 6$, then $\Delta \leq r_{2} / 2$; if $1 \leq k \leq 9$, then $\Delta \leq r_{2} / 2 \sqrt{2}$; and if $1 \leq k \leq 11$, then $\Delta \leq r_{2} / 3$.

Let the domain $G$ be an equilateral triangle $G_{t}$. Then, we construct an oblique grid on $G_{t}$ as was described above. Let $s$ be a vertex of the triangle $G_{t}$; therefore, $s$ coincides with a point of $T_{\Delta}$. Then, in the case $r_{2}=\Delta$ the set $G_{t} \cap R(s)$ contains three different points of $T_{\Delta}$; for $r_{2}=\sqrt{3} \Delta, G_{t} \cap R(s)$, contains four different points of $T_{\Delta}$, etc. For any other point $s$ in $T_{\Delta}$ (that is not a vertex of the triangle), the number of different points of $T_{\Delta}$ in $G_{t} \cap R(s)$, is no less than this number in the case when $s$ is a vertex of the triangle. Therefore, if $1 \leq k \leq 3$, then $\Delta \leq r_{2}$; if $1 \leq k \leq 4$, then $\Delta \leq r_{2} / \sqrt{3}$; if $1 \leq k \leq 6$, then $\Delta \leq r_{2} / 2$; if $1 \leq k \leq 8$, then $\Delta \leq 2 r_{2} / 3 \sqrt{3}$; and if $1 \leq k \leq 10$, then $\Delta \leq r_{2} / 3$.

As a result, we have relations between the covering multiplicity, the grid step size, the radius of the covering circles, and the domain $G$. If the sufficient conditions formulated above are satisfied, then condition (1) is also satisfied; therefore, P3 is solvable.

\section{Consider P2.}

Let the grid step $\Delta$ be chosen, and the rectangular grid and the set $T_{\Delta}$ be constructed. By solving problem W1, we obtain a $k$-covering of the set $T_{\Delta}$ for which the total area of the circles covering $T_{\Delta}$ is minimal. If $T_{\Delta}$ is covered with circles of the given radii $r_{1}$ and $r_{2}$, it is not guaranteed that the given set $G$ is completely covered. The grid with the step $\Delta$ on the set $G$ generates squares with the diagonal equal to $\Delta \sqrt{2}$. Let $r_{2}>\Delta \sqrt{2}$. If we decrease the radii $r_{1}$ and $r_{2}$ by the half of this diagonal when solving the P3, then the circles of the original radii $r_{1}$ and $r_{2}$ for the arrangement of circles obtained in this problem certainly cover the entire set $G$. Therefore, an approximate solution of P2 can be obtained by solving P3 for $T_{\Delta}$ with the circle radii decreased by $\alpha=$ $\Delta \sqrt{2} / 2$, and then again increasing the radii of the found circles to $r_{1}$ and $r_{2}$. The sufficient solvability conditions for $\mathrm{P} 2$ are the same as for the solvability of P3 with $r_{2}$ replaced by $r_{2}-\alpha$. Thus, to check the solvability of P2 and P3, we may check conditions (1) or obtain the sufficient conditions as described above.

Below, we use the notation $m_{1} / m_{2}$ to indicate covering with $m_{1}$ circles of radius $r_{1}$ and $m_{2}$ circles of radius $r_{2}$.

Numerous computational experiments show that the less the grid step size $\Delta$ is, the closer the result to the optimal covering. For example, when the unit square $Q$ is covered with circles of radii 0.55 and 0.2 for $\Delta$ equal to $0.1,0.05$, $0.025,0.0125$, and 0.01 , the density of the 1-covering of $Q$ is, respectively, $2.654,1.829,1.760,1.704$, and 1.454 . The covering densities were obtained for the circle numbers equal, respectively, to $2 / 6,1 / 7,0 / 14,1 / 6$ and $1 / 4$. Thus the choice of the value $\Delta$ is very important to obtain an acceptable solution of the covering problem.

The approximate solution of $\mathrm{P} 2$ can be considered as an approximate solution of P1. 


\section{Let us consider the problem P5 then P4.}

The solution of problem $\mathrm{P} 5$ is reduced to the solution of problem (5)-(6) with the following constraint:

$$
z_{i} \in Z^{+}, 1 \leq i \leq 2 n \text {, where } Z^{+}=\{0,1,2,3, \ldots\} .
$$

As a result, we have an integer linear programming (ILP) problem (5), (6) and (8) of size $2 n$. Denote this problem by W2.

Let $T_{\Delta}, T_{\Delta} \neq \emptyset$, be already constructed. Then one can easily see that system W2 is solvable and, consequently, so is $\mathrm{P} 5$.

For ensuring the solvability of $\mathrm{P} 4$, we require that $r_{2}>2 \alpha, \alpha=\Delta \sqrt{2} / 2$. For solving P4, we introduce new circle radii $r_{1}-\alpha$ and $r_{2}-\alpha$ and solve $\mathrm{P} 5$ with them. We treat locations of centers of the mentioned circles in the solution of $\mathrm{P} 5$ as an approximate solution to $\mathrm{P} 4$, assuming that circle radii again are equal to initial values $r_{1}$ and $r_{2}$, correspondingly.

Thus we see that for solving $\mathrm{P} 4$ it is necessary to solve $\mathrm{P} 5$. We treat the solution to $\mathrm{P} 4$ as an approximate solution to $\mathrm{P} 1$. Therefore, one can solve P1 by solving problem $\mathrm{W} 1$, where $z_{i} \in\{0,1\}, 1 \leq i \leq 2 n$, or by solving problem W2, where $z_{i} \in Z^{+}, 1 \leq i \leq 2 n$. Later in Section 5 we establish that these solutions can differ.

For formulation W1, a heuristic algorithm described in [26] solves P3 and P2, and, consequently, P1, even when the problem dimension is as large as 14000 . Note that without the use of this algorithm, we can solve the mentioned problems with no more than 5000 variables (moreover, in certain cases, the maximum number of variables guaranteeing the problem solvability is only 2000 ). The heuristic algorithm essentially uses the fact that problem variables are Boolean. Unfortunately, the authors have failed to construct a similar algorithm for solving problem W2 with $z_{i} \in Z^{+}$, because the reduction of a general integer linear programming problem to a Boolean linear programming problem is a rather cumbersome task.

\section{Constraints on the Distances Between the Circle Centers}

In some cases, it is important that the centers of the covering circles are all different. For example, when navigation systems are designed, each point may contain no more than one device. Let us introduce the restrictions on the minimum distance between the centers of covering circles in the same way as it was done in $[25,26]$. Note that for the covering problems, various restrictions were introduced besides coverage conditions. For example, in [34-36].
Suppose that it is required that the distance between the centers of the circles of radius $r_{1}$ must be at least $q_{1}$. For each point $t_{i}$ in $T_{\Delta}=\left\{t_{1}, t_{2}, \ldots, t_{n}\right\}$, let $p_{i}$ be the number of points $t_{j}$ in $T_{\Delta}(j \neq i)$ for which $d\left(t_{i}, t_{j}\right)<q_{1}$. Define the coefficients:

$$
\begin{aligned}
& b_{i j}=\left\{\begin{array}{l}
1 \quad \text { if } \quad d\left(t_{i}, t_{j}\right)<q_{1}, \\
0 \quad \text { if } \quad d\left(t_{i}, t_{j}\right) \geq q_{1},
\end{array}\right. \\
& i \neq j, \quad 1 \leq i, j \leq n ; \quad b_{i i}=p_{i}, \quad 1 \leq i \leq n .
\end{aligned}
$$

Now the condition that the distances between the centers of the circles of radius $r_{1}$ must be at least $q_{1}$ is written as (see $[25,26])$ :

$$
\begin{gathered}
b_{11} z_{1}+b_{12} z_{2}+\ldots+b_{1 n} z_{n} \leq p_{1}, \\
\ldots \\
b_{n 1} z_{1}+b_{n 2} z_{2}+\ldots+b_{n n} z_{n} \leq p_{n} .
\end{gathered}
$$

If the problem $\mathrm{W} 1$ is solved subject to the additional constraints (9), then we obtain the desired covering in which the centers of the covering circles of radius $r_{1}$ are at the distance at least $q_{1}$.

Let it be required that the distance between the centers of the circles of radius $r_{2}$ is not less than a given number $q_{2}$. Let $f_{i}$ and $c_{i j}$ be determined in the same way as $p_{i}$ and $b_{i j}(1 \leq i, j \leq n)$ only using $q_{2}$ instead of $q_{1}$. Then, the conditions that the distance between the centers of the circles of radius $r_{2}$ is not less than the given number $q_{2}$ take the form:

$$
\begin{gathered}
c_{11} z_{n+1}+c_{12} z_{n+2}+\ldots+c_{1 n} z_{2 n} \leq f_{1}, \\
\ldots \\
c_{n 1} z_{n+1}+c_{n 2} z_{n+2}+\ldots+c_{n n} z_{2 n} \leq f_{n} .
\end{gathered}
$$

Furthermore, suppose that it is required that the distance between the centers of the circles of radii $r_{1}$ and $r_{2}$ must be not less than a given number $q_{3}$. Let $g_{i}$ and $e_{i j}(1 \leq i, j \leq n)$ be determined in the same way as $p_{i}$ and $b_{i j}$ but using $q_{3}$ instead of $q_{1}$. We also define $h_{i, n+i}=g_{i}$ and $h_{i j}=0$ for $1 \leq i \leq n, n+1 \leq j \leq 2 n$, and $j \neq i$. Then, the conditions that the distance between the centers of the circles of radii $r_{1}$ and $r_{2}$ is not less than the given number $q_{3}$ have the form (see [25]):

$$
\begin{aligned}
& \left(\begin{array}{cccccc}
e_{11} & \ldots & e_{1 n} & h_{i, n+1} & \ldots & 0 \\
e_{21} & \ldots & e_{2 n} & 0 & \ldots & 0 \\
& & & \ldots & & \\
e_{n 1} & \ldots & e_{n n} & 0 & \ldots & h_{i, n+n}
\end{array}\right) \\
& \times\left(\begin{array}{c}
z_{1} \\
z_{2} \\
\ldots \\
z_{2 n}
\end{array}\right) \leq\left(\begin{array}{c}
g_{1} \\
g_{2} \\
\ldots \\
g_{n}
\end{array}\right)
\end{aligned}
$$


If $q_{1}=q_{2}=q_{3}$, then we set $p_{i}=f_{i}=g_{i}$ for $1 \leq i \leq n$ and $b_{i j}=c_{i j}=e_{i j}$ for $1 \leq i, j \leq n$ in constraints (10) and (11). Constraints (9), (10) and (11) may be imposed simultaneously or one may impose only those of them that are needed in a specific problem. In each case, we obtain a ILP.

If constraints on the distance between the circle centers are imposed, the problem can be unsolvable. For example, for the problem of finding a 3-covering of an equilateral triangle with the side $c$, it is senseless to require that the distance between the circle centers is greater than $c$ because in this case the circle centers will be outside the triangle.

\section{Finding Lower Bounds on the Density of Covering for Specific}

\section{Cases}

In [26] one obtains approximate lower bounds for the density of the $k$-covering of the set $G$ with circles of radii $r_{1}$ and $r_{2}$, provided that the minimum distance between centers of covering circles is not less than $\lambda, \lambda \geq 3 \Delta$, and the covering multiplicity satisfies the condition $1 \leq k \leq 4$.

In this paper, we obtain approximate lower bounds for densities of coverings with any multiplicity $k \geq 1$, imposing no constraint on the minimum distance between centers of covering circles.

Problem P1 allows a $k$-covering such that the total area of covering circles is minimal (an optimal $k$-covering). Let an optimal covering contain $m$ circles of given radii. Denote centers of these circles by $c_{1}, c_{2}, \ldots, c_{m}$. We use subscripts for circle centers even when their locations coincide. Since the set $G$ is convex, the center of each covering circle belongs to $G$. The definition of a $k$-covering implies that for any point $s \in G$ there exists at least $k$ centers $c_{j}$ among $c_{1}, c_{2}, \ldots, c_{m}$ such that $d\left(s, c_{j}\right) \leq \max \left\{r_{1}, r_{2}\right\}$.

Choose a grid step $\Delta, \Delta>0$, and construct the set $T_{\Delta}=\left\{t_{1}, \ldots, t_{n}\right\}, t_{i} \in G, 1 \leq i \leq n$. By construction of the set $T_{\Delta}$, the distance between any point $s \in G$ and the nearest to its point in $T_{\Delta}$ does not exceed $\alpha=\Delta \sqrt{2} / 2$.

If a circle center $c_{i}$ coincides with no point in $T_{\Delta}$, then we move this center to the nearest point $t_{j} \in T_{\Delta}$. We move all circle centers in the mentioned way.

In addition to point shifts, we increase the radii of the covering circles by $\alpha$. The set of circles of radii $r_{1}$ and $r_{2}$ centered in $c_{1}, c_{2}, \ldots, c_{m}$ forms the desired optimal covering of $G$. Suppose that this set consists of $n_{1}^{0}$ circles of radius $r_{1}$ and $n_{2}^{0}$ circles of radius $r_{2}$. Then, the density of the optimal covering is $P^{0}\left(r_{1}, r_{2}\right)=\left(n_{1}^{0} r_{1}^{2}+n_{2}^{0} r_{2}^{2}\right) \tau$, where $\tau=\pi / S(G)$ and $S(G)$ is the area of the set $G$.
After the circle centers have been shifted to the points of $T_{\Delta}$ and their radii have been increased by $\alpha$ they form a $k$-covering of $G$ and, therefore, a $k$-covering of $T_{\Delta}$. Define $P_{\alpha}^{\#}=\left(n_{1}^{0}\left(r_{1}+\alpha\right)^{2}+n_{2}^{0}\left(r_{2}+\alpha\right)^{2}\right) \tau$. Solve P5 of $k$-covering of $T_{\Delta}$ with circles of radii $r_{1}+\alpha$ and $r_{2}+\alpha$. As a result, we obtain $n_{1}$ circles of radius $r_{1}+\alpha$ and $n_{2}$ circles of radius $r_{2}+\alpha$. It is important that $n_{1}$ and $n_{2}$ are known in contrast to the unknown quantities $n_{1}^{0}$ and $n_{2}^{0}$. Define $P_{\alpha, o p t}^{\#}=\left[n_{1}\left(r_{1}+\right.\right.$ $\left.\alpha)^{2}+n_{2}\left(r_{2}+\alpha\right)^{2}\right] \tau$. It is clear that $P_{\alpha}^{\#}-P_{\alpha, o p t}^{\#} \geq 0$.

Let $m_{1}=n_{1}^{0}-n_{1}$ and $m_{2}=n_{2}^{0}-n_{2}$. Then the inequality $P_{\alpha}^{\#}-P_{\alpha, o p t}^{\#} \geq 0$ implies

$$
m_{1}\left(r_{1}+\alpha\right)^{2}+m_{2}\left(r_{2}+\alpha\right)^{2} \geq 0
$$

Define the new quantity $P_{*}^{\#}\left(r_{1}, r_{2}\right)=\left(n_{1} r_{1}^{2}+n_{2} r_{2}^{2}\right) \tau$, where $n_{1}$ and $n_{2}$ are found by solving P5. For brevity, set $P N$ $=P_{\star}^{\#}\left(r_{1}, r_{2}\right)$. We will examine the difference $B=P^{0}\left(r_{1}, r_{2}\right)$ $P_{*}^{\#}\left(r_{1}, r_{2}\right)=\left(m_{1} r_{1}^{2}+m_{2} r_{2}^{2}\right) \tau$ for various possible values of $m_{1}$ and $m_{2}$.

(a) If $m_{1}=m_{2}=0$, then $n_{1}^{0}=n_{1}, n_{2}^{0}=n_{2}$ and $B=0$.

(b) If $m_{1}=0$ or $m_{2}=0$, then (12) implies that either $m_{2} \geq 0$ or $m_{1} \geq 0$, respectively, and $B \geq 0$; therefore,

$$
P_{\star}^{\#}\left(r_{1}, r_{2}\right) \leq P^{0}\left(r_{1}, r_{2}\right)
$$

(c) Consider the case $m_{1}>0$, when the number of large circles decreases by a number $p(p>0)$ after solving P5. The number of small circles changes by a $q, n_{2}=$ $n_{2}^{0}+q(q \leq 0$ or $q>0)$. In this case, (12) implies $\left[p\left(r_{1}+\alpha\right)^{2}-q\left(r_{2}+\alpha\right)^{2}\right] \geq 0$. Then $B=\left[p r_{1}^{2}-q r_{2}^{2}\right] \tau$. If $q \leq$ 0 , then it is clear that $B \geq 0$, and inequalities (13) hold. Let $q>0$. Then $q \leq p\left(\frac{r_{1}+\alpha}{r_{2}+\alpha}\right)^{2}$. If we assume $B<0$ in this case, then $p r_{1}^{2}-q r_{2}^{2}<0$ and $q>p \frac{r_{1}^{2}}{r_{2}^{2}}$. Inequalities $q \leq p\left(\frac{r_{1}+\alpha}{r_{2}+\alpha}\right)^{2}$ and $q>p \frac{r_{1}^{2}}{r_{2}^{2}}$ cannot simultaneously be true for any $\alpha \geq 0$. Hence, the assumption $B<0$ does not hold, we have $B \geq 0$, and inequality (13) holds.

(d) Consider the case $m_{1}<0$, when the number of large circles increases by a number $p>0$ after solving P5. If the number of large circles did not change, then $m_{2}=0$, and (b) implies that inequality (13) holds. The number of circles of radius $r_{1}$ and the number of circles of radius $r_{2}$ cannot increase simultaneously. Suppose that when the number of large circles increased, the number of small circles decreased by $q>0$. Then, inequality (12) implies

$$
\begin{aligned}
& \left(n_{1}^{0}-\left(n_{1}^{0}+p\right)\right)\left(r_{1}+\alpha\right)^{2} \\
& +\left(n_{2}^{0}-\left(n_{2}^{0}-q\right)\right)\left(r_{2}+\alpha\right)^{2} \\
& =-p\left(r_{1}+\alpha\right)^{2}+q\left(r_{2}+\alpha\right)^{2} \geq 0 .
\end{aligned}
$$


Let $B<0$. Then $-p r_{1}^{2}+q r_{2}^{2}<0$. Taking into account (14), we obtain two inequalities

$$
p>q\left(\frac{r_{2}}{r_{1}}\right)^{2}, \quad p \leq q\left(\frac{r_{2}+\alpha}{r_{1}+\alpha}\right)^{2}
$$

where $p$ and $q$ are integers greater than zero. In the case under consideration $n_{1}=n_{1}^{0}+p$; then $p=n_{1}-n_{1}^{0}$ and, therefore, $p \leq n_{1}$. If $n_{1}=0$, then $p>0$ does not exist and the assumption $B<0$ does not hold; therefore, $B \geq 0$ and we have (13). Now consider the case $n_{1}>0, p>0$. Assume that both inequalities in (15) hold; i.e., for a certain $q, p$ is within the interval $I_{q}=\left(q\left(\frac{r_{2}}{r_{1}}\right)^{2}, q\left(\frac{r_{2}+\alpha}{r_{1}+\alpha}\right)^{2}\right]$. In this case $B<0$, and no lower bound for $P^{0}\left(r_{1}, r_{2}\right)$ is found. If such $p$ and $q$ do not exist, then the assumption $B<0$ does not hold, and we have (13).

Thus, in some cases we have not found a lower bound for $P^{0}\left(r_{1}, r_{2}\right)$ (both inequalities in (15) hold). If inequalities (15) do not hold, then the assumption $B<0$ is not true and inequality (13) holds.

Consider some particular cases of the covering. Notice that unlike [26] where the P3 is studied, here for solving $\mathrm{P} 5$ it is not necessary to calculate the minimum distance between centers of covering circles that was mentioned above.

Let $Q$ be a unit square and it is required to cover it with circles of radii $r_{1}=0.55$ and $r_{2}=0.30$. Choose $\alpha=0.01$ and $\Delta=0.01 \sqrt{2}$. By solving P5, we obtain $n_{1}=0$ and $n_{2}=$ 6. Then it is clear that $m_{1} \geq 0$. According to (b), for $m_{1}=0$ we obtain (13); for $m_{1}>0$, (c) implies (13). As a result, the lower bound for $P^{0}(0.55,0.30)$ is $P_{\star}^{\#}(0.55,0.30)=1.696$.

Consider one more particular case. Suppose that we want to find a 2-covering of $Q$ with circles of radii $r_{1}=0.55$ and $r_{2}=0.25$. By setting $\alpha=0.0125, \Delta=0.0125 \sqrt{2}$, and solving P5, we obtain $n_{1}=0$ and $n_{2}=16$. Next, we obtain the lower bound for the density of the 2-covering equal to 3.141. If we set $\alpha=0.01$ and $\Delta=0.01 \sqrt{2}$ instead of $\alpha=0.0125$ and $\Delta=0.0125 \sqrt{2}$, then by solving P5 we obtain $n_{1}=1$ and $n_{2}=12$. Next, using the interval $I_{1}=$ $(0.20661,0.21556]$, we find, as in the second particular case, that inequality (13) holds, and the lower bound for the density of the 2-covering is 3.306. This bound is greater than the earlier bound 3.141; therefore, we improved the bound by decreasing $\alpha$ and $\Delta$.

\section{Numerical Results}

Consider a covering of a rectangular $(1.22 \times 0.82)$ set $R$. Evidently, its area is close to 1 (more precisely, $1.22 \times 0.82=$ 1.0004). Let the radii of covering circles $r_{1}=0.58$ and $r_{2}=$ 0.46 and let the covering multiplicity be 2 . Using problem W1 with $\Delta=0.02$, we conclude that the set $R$ can be covered with three circles of the radius $r_{1}$ and one circle of the radius $r_{2}$, while the density of the 2-covering equals 3.835 . Using problem W2 with the same grid step $\Delta$, we conclude that the set $R$ can be twice covered with two circles of the radius $r_{1}$ with coinciding centers and two circles of the radius $r_{2}$ whose centers also coincide. We found that the density of this covering is 3.443. Therefore, problems $\mathrm{W} 1$ and $\mathrm{W} 2$ give different results with the same initial data. See Figure 1(a) and Figure 1(b) for the mentioned coverings of the set $R$ obtained with the help of problems W1 and W2.

Using problem W2 with the grid step $\Delta=0.02$, we conclude that the set $R$ can be twice covered with two circles of the radius $r_{1}$ with coinciding centers and two circles of the radius $r_{2}$ whose centers also coincide, consequently $n_{1}=2$ and $n_{2}=2$. For the case $m_{1} \geq 0$ according (a)-(c)n from section 4 we obtain (13). It can be shown that for the case under consideration $m_{1}<0$ is excluded. As a result, the lower bound for $P^{0}(0.58,046)$ is $P_{*}^{\#}(0.58,0.46)=3.443$.

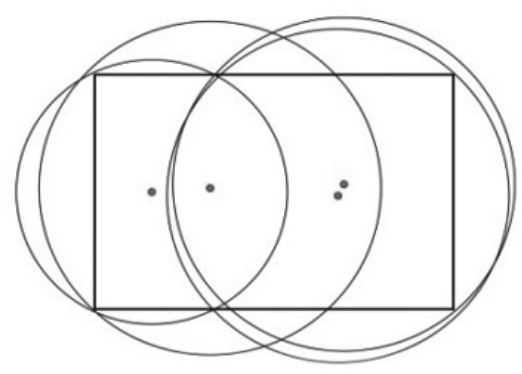

(a)

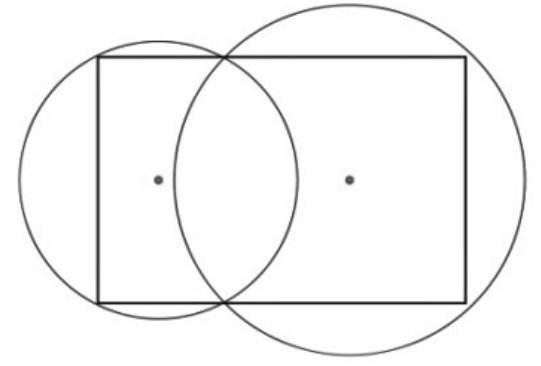

(b)

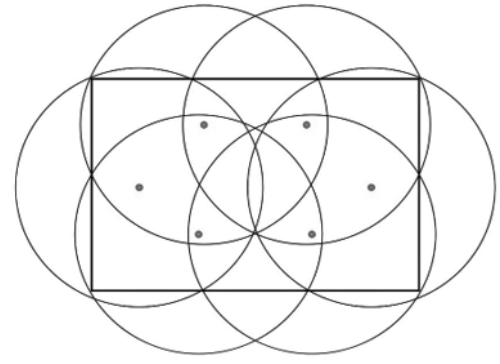

(c)

Figure 1: Covering of a rectangle $(1.22 \times 0.82)$ with the circles of radii $r_{1}=0.58$ and $r_{2}=0.46$ a) 2-covering is obtained by problem W1, b) 2 -covering is obtained by problem $\mathrm{W} 2, \mathrm{c}) 2$-covering is obtained by problem $\mathrm{W} 1$ with restriction $\min \left[d\left(c_{i}, c_{j}\right) \geq \lambda: 1 \leq i, j \leq m, i \neq j\right]$, where $\lambda=0.6 r_{2}$. 
We performed corresponding computations for covering $R$ with various $r_{1}$ and $r_{2}$, the results are presented in Table 1 . The problem P5 for obtaining the lower bounds of the covering density was solved exactly without using heuristics. As has been mentioned above the lower bound on the covering density is not guaranteed to be found. However, no such cases were encountered in our computations.

In the Figure 1(c) we see the 2-covering of $R$ with circles of radii $r_{1}=0.58$ and $r_{2}=0.46$ obtained under the additional requirement that the minimum distance between centers of covering circles (that was mentioned above) should be not less than $0.6 r_{2}$. This covering is performed with six circles of the radius $r_{2}$ so that the covering density equals 3.989 .

Let us now consider other covering of the rectangle $R$ with circles of radii $r_{1}$ and $r_{2}$. Its results are shown in Table 1. See [26] for analogous results for the square covering problem and to compare with some well-known publications on this topic. Results given in Table 1 are new, so we can compare them with no other ones. We can only estimate these results, based on approximate lower bounds for the covering density and the calculated values of the density. In certain cases, lower bounds and obtained values coincide (see Table 1), so the coverings are unimprovable. In the rest of cases, the difference between obtained values and approximate lower bounds for the covering density characterizes the acceptability (quality) of the covering.

The results from Table 1 were obtained using a rectangular grid. For studying the dependence of the density on grid properties, we have covered the rectangle with rectangular and oblique grid. In these calculations the same value step size $\Delta$ were selected. We obtained the number of circles for covering with circles of radii 0.55 and 0.30 using an oblique grid. We got the number of circles and therefore the covering densities coinciding with those given in Table 1. Corresponding coincidences were obtained for the remaining values of $r_{1}$ and $r_{2}$ of Table 1 when using oblique and rectangular grids. Thus, in the considered cases no influence of the grid type was revealed. As noted in section 2 (and the example is given) the choice of the value $\Delta$ is important.

If we are not satisfied with the covering quality, we try to improve the obtained value and (or) lower bound of the covering density. This can be done by repeatedly lessening the value $\Delta$ and solving problems $\mathrm{P} 4$ and $\mathrm{P} 5$, provided that the computer capabilities allow one to do this.

The computations were performed on an Intel Core i73537U computer with $6 \mathrm{~Gb}$ of memory and clock rate of 2.5 $\mathrm{GHz}$ under Windows 10 . The computation time for each of the results discussed above was 1-3 minutes. Since the covering problems in many applications are solved in advance rather than in the online mode, such a computation time is quite acceptable.

For the investigation of the ILPs problems constructed in this work, subgradient and other methods can be used. It is important that the approach proposed in this paper is simple and effective. The numerical results confirm the effectiveness of the method for various versions of $k$-covering problems.

Table 1: Covering of a rectangle $(1.22 \times 0.82)$ for given radii and multiplicity of the covering

\begin{tabular}{lcccc}
\hline Radii of circles $r_{1} / r_{2}$ & \multicolumn{4}{c}{ Multiplicity of covering $k$} \\
\cline { 2 - 5 } & $\begin{array}{c}\text { Approximate lower } \\
\text { bounds (PN) of } \\
\text { covering density and } \\
\text { corresponding } \\
\text { number of circles }\end{array}$ & $\begin{array}{c}\text { Obtained covering } \\
\text { density and } \\
\text { corresponding } \\
\text { number of circles }\end{array}$ & $\begin{array}{c}\text { Approximate lower } \\
\text { bounds (PN) of } \\
\text { covering density and } \\
\text { corresponding }\end{array}$ & $\begin{array}{c}\text { Obtained covering } \\
\text { density and } \\
\text { corresponding } \\
\text { number of circles } \\
\text { number of circles }\end{array}$ \\
\hline $0.55 / 0.30$ & $1.516(1 / 2)$ & $1.696(0 / 6)$ & $3.031(2 / 4)$ & $3.392(0 / 12)$ \\
\hline $0.55 / 0.25$ & $1.539(1 / 3)$ & $1.767(0 / 9)$ & $2.945(0 / 15)$ & $3.338(0 / 17)$ \\
\hline $0.55 / 0.20$ & $1.382(0 / 11)$ & $1.703(1 / 6)$ & $2.765(0 / 22)$ & $3.266(0 / 26)$ \\
\hline $0.45 / 0.30$ & $1.696(0 / 6)$ & $1.696(0 / 6)$ & $3.252(2 / 7)$ & $3.392(0 / 12)$ \\
\hline $0.45 / 0.25$ & $1.571(0 / 8)$ & $1.767(0 / 9)$ & $2.945(0 / 15)$ & $3.338(0 / 17)$ \\
\hline $0.45 / 0.20$ & $1.382(0 / 11)$ & $1.641(1 / 8)$ & $2.765(0 / 22)$ & $3.267(0 / 26)$ \\
\hline $0.35 / 0.30$ & $1.696(0 / 6)$ & $1.696(0 / 6)$ & $3.032(2 / 8)$ & $3.236(4 / 6)$ \\
\hline $0.35 / 0.25$ & $1.539(4 / 0)$ & $1.742(3 / 3)$ & $2.906(5 / 5)$ & $3.109(4 / 8)$ \\
\hline
\end{tabular}




\section{Conclusion}

A numerical method for investigating $k$-coverings of a convex bounded set with circles of two given radii is proposed. Cases with constraints on the distances between the covering circle centers are considered. An algorithm for finding an approximate number of such circles and the arrangement of their centers is described. For certain specific cases, approximate lower bounds of the density of the $k$-covering of the given domain are found. We use 0-1 linear programming or general integer linear programming models. $\mathrm{Nu}-$ merical results demonstrating the effectiveness of the proposed methods are presented.

The proposed method with constructing a grid on $G$ can be extended for covering problems in spaces of three and more dimensions. While solving the problem of covering with circles of equal radii, we obtain an ILPs of dimension $n \times 2 n$, where $n$ is the number of nodes in the grid on $G$. In the case of covering $G$ with balls of $s$ different (fixed) radii, the dimension of the ILP is $(s \times n) \times 2 n$ without taking into account constraints on the distances between the ball centers. The solution of such problems requires either longer computation time or other methods for solving large-scale ILPs.

Acknowledgement: Authors express their gratitude to the anonymous referees for the remarks and useful recommendations which allowed to improve this article.

\section{References}

[1] Brusov V.S., Piyavskii S.A., A computational algorithm for the optimal covering of planar domains, USSR Computational Mathematics and Mathematical Physics, 1971, 11(2), 17-27

[2] Drezner Z., Facility location: A survey of applications and methods, Springer-Verlag, New York, 1995

[3] Drezner Z., Hamacher H.W., Facility location: Applications and theory, Springer-Verlag, Berlin, Germany, 2002

[4] ReVelle C.S., Eiselt H.A., Location analysis: A synthesis and survey, European Journal of Operational Research, 2005, 165, 1-19

[5] Zanjirani Farahani R., Hekmatfar M., Facility Location: Concepts, Models, Algorithms and Case Studies, Physica-Verlag Heidelberg, 2009

[6] Berman O., Drezner Z., Krass D., Wesolowsky G.O., The variable radius covering problem, European Journal of Operational Research, 2009, 196, 516-525

[7] Ammari Y.M., Challenges and Opportunities of Connected kCovered Wireless Sensor Networks, Springer, Berlin, 2009

[8] Huang C.F., Tseng Y.C., A Survey of Solutions to the Coverage Problems in Wireless Sensor Networks, Journal of Internet Technology, 2005, 6(1), 1-8
[9] Wang B., Coverage Problems in Sensor Networks: A Survey, ACM Computing Surveys, 2011, 43(4), 167-170, DOI: 10.1145/1978802.1978811

[10] Yeasmin N., k-Coverage Problems and Solutions in Wireless Sensor Networks: A Survey, International Journal of Computer Applications, 2014, 100(17), 1-6, DOI: 10.5120/17614-8309

[11] Astrakov S.N., Erzin A.I., Zalyubovskii V.V., Sensor networks and planar region coverage with circles, Discrete analysis and operation study, 2009, 16(3), 3-19

[12] Kumar S., Lai T.H., Balogh J., On k-coverage in a mostly sleeping sensor network, In Proceedings of the 10th Annual International Conference on Mobile Computing and Networking MobiCom'04, 2004, 144-158, DOI: 10.1145/1023720.1023735

[13] Nurmella K.J., Conjecturally optimal coverings of an equilateral triangle with up to 36 equal circles, Experimental Mathematics, 2000, 9(2), 241-250, DOI: 10.1080/10586458.2000.10504649

[14] Nurmella K.J., Covering a circle by congruent circular discs (preprint), Departament of Computer Sciences and Engineering, Helsinki University of Technology, 1998

[15] Nurmella K.J., Östergard, P.R.J.: Covering a square with up to 30 equal circles. In.: Research report A62 Laboratory for Technology Helsinki University, 2000

[16] Suzuki A., Drezner, Z. The minimum number equitable radius location problems with continuous demand, European Journal of Operational Research, 2009, 195, 17-30, DOI: 10.1016/j.ejor.2008.01.022

[17] Erzin A., Astrakov S., Min-Density Stripe Covering and Applications in Sensor Network, In: Murgante B., Gervasi O., Iglesias A., Taniar D., Apduhan B.O. (eds.) ICCSA 2011. LNCS, Springer Heidelberg, 2011, 6784, 152-162, DOI: 10.1007/978-3-642-21931-3_13

[18] Astrakov S.N., Erzin A.I., Construction of efficient covering models in the monitoring of extended objects, Vychisl. Tekhnol., 2012, 17(1), 26-34

[19] Erzin A.I., Shabelnikova N.A., About density of a covering of a strip with identical sectors, J. Appl. Industr. Math., 2015, 9(4), 461-468

[20] Fejes Tóth, G., Multiple packing and covering of the plane with circles. Acta Mathematica Academiae Scientiarum Hungarica, 1976, 27(1-2), 135-140

[21] Galiev Sh.I., Khorkov A.V., Multiple circle coverings of an equilateral triangle, square, and circle. Diskretn. Analiz Issl. Oper., 2015, 22(6), 5-28, DOI: 10.17377/daio.2015.22.482

[22] Chakrabarty K., lyengar S.S, Qi H., Cho E., Grid coverage for surveillance and target location in distributed sensor networks, IEEE Transactions on Computers, 2002, 51(12), 1448-1458

[23] Fejes Tóth, G., Covering the plane with two kinds of circles, Discrete and Computational Geometry, 1995, 13(3-4), 445-457, DOI: 10.1007/BF02574055

[24] Galiev Sh.I., Karpova M.A., Optimization of Multiple Covering of a Bounded Set with Circles, Computational Mathematics and Mathematical Physics, 2010, 50, 721-732

[25] Galiev Sh.I., Lisafina M.S., Linear models for the approximate solution of the problem of packing equal circles into a given domain, European Journal of Operational Research, 2013, 230, 505-514

[26] Galiev Sh.I., Khorkov A.V., Optimization of the Number and Arrangement of Circles of Two Radii for Forming a k-Covering of a Bounded Set, Computational Mathematics and Mathematical Physics, 2019, 59(4), 676-687 
[27] Galiev Sh.I., Khorkov A.V., On the Number and Arrangement of Sensors for Multiple Covering of Bounded Plane Domains, Journal of Applied and Industrial Mathematics, 2019, 13(1), 43-53

[28] Garey M.R., Johnson D.S., Computers and Intractability: A Guide to the Theory of NP-completeness, W. H. Freeman and Co. New York, NY, USA, 1979

[29] Megiddo N., Supowit K., On the complexity of some common geometric location problems, SIAM Journal on Computing, 1984, 13(1), 182-196 10.1137/0213014

[30] Culberson J.C., Reckhow R.A., Covering polygons is hard. J. Algorithms, 1994, 17, 2-44

[31] Kuzyurin, N.N., On the complexity of asymptotically optimal coverings and packings, Doklady Mathematics, 1998, 58(3), 345346

[32] Eremeev A.V., Zaozerskaya L.A., Kolokolov A.A., Set covering problem: Complexity, algorithms, and experimental studies, Diskretn. Analiz Issl. Oper., 2009, 7(2), 22-46
[33] Khachai M.Yu., Poberii M.I., The computational complexity and approximability of a series of geometric covering problems, Trudy Inst. Mat. i Mekh. UrO RAN, 2012, 18(3), 247-260

[34] Astrakov S.N., Coverings of Sets with Restrictions on the Arrangement of Circles, In: Yu. G. Evtushenko, M. Yu. Khachay, O. V. Khamisov, Yu. A. Kochetov, V.U. Malkova, M.A. Posypkin (eds.): Proceedings of the OPTIMA-2017 Conference, Petrovac, Montenegro, 2017, 67-72

[35] Doğançay K., Hmam H., Optimal Angular Sensor Separation for AOA Localization, Signal Process, 2008, 88(5), 1248-1260

[36] Kim J.E., Yoon M.K., Han J., Lee C.G., Sensor Placement for 3Coverage with Minimum Separation Requirements, Distributed Computing in Sensor Systems: 4th IEEE International Conference, 2008, 5067, 266-281, DOI: 10.1007/978-3-540-69170-9_18 